\title{
OIKOS
}

\section{Vertical relief facilitates spatial segregation of a high density large carnivore population}

\begin{tabular}{|c|c|}
\hline Journal: & Oikos \\
\hline Manuscript ID & OIK-06724.R1 \\
\hline Wiley - Manuscript type: & Research \\
\hline Keywords: & $\begin{array}{l}\text { auto-correlated Kernel density estimation, elevation gradient, } \\
\text { intraspecific competition, planimetric, predation pattern, topography }\end{array}$ \\
\hline Abstract: & $\begin{array}{l}\text { Quantifying the distribution and size of home ranges is critical for } \\
\text { understanding animal spatial dynamics. This is particularly important for } \\
\text { large carnivores that reside in fragmented landscapes. Most studies that } \\
\text { estimate home range consider only a bivariate frequency distribution } \\
\text { represented by a two-dimensional planimetric surface. The underlying } \\
\text { assumption of these approaches is that the animals inhabit landscapes } \\
\text { that are completely flat. Of course, this is rarely the case. Here we } \\
\text { investigated the influence of vertical relief and three-dimensional } \\
\text { landscape features on the home range patterns of a high density } \\
\text { carnivore. Via GPS telemetry-tracking of a population of Persian leopards } \\
\text { (Panthera pardus saxicolor, n=6), and globally-available digital elevation } \\
\text { models (DEMs), we calculated the surface area of home ranges in } \\
\text { comparison to traditional planimetric estimates. We also investigated } \\
\text { predation patterns of leopards across elevation gradients using GPS } \\
\text { location data and kill site analysis. The topographic measurements } \\
\text { exceeded planimetric estimates by up to } 38 \% \text { which suggests that } \\
\text { planimetric modeling underestimates home range size, particularly when } \\
\text { animals inhabit variable terrain. We also observed that resident leopards } \\
\text { exhibit significant altitudinal partitioning of predation, suggesting that } \\
\text { leopards that have overlapping home ranges may still utilize exclusive } \\
\text { hunting territories. We discuss the ways in which planimetric approaches } \\
\text { may be underestimating aspects of animal ranging behavior and ecology. } \\
\text { We conclude that topography should be considered, not as an ancillary } \\
\text { metric, but as an important aspect of home range calculation. Our } \\
\text { approach can enhance understanding of spatial requirements, population } \\
\text { density, intra-guild sympatric competition and conflict management of } \\
\text { large felids inhabiting rugged landscapes. }\end{array}$ \\
\hline
\end{tabular}

\section{SCHOLARONE" Manuscripts}


1 Vertical relief facilitates spatial segregation of a high density large carnivore population

2

3

4

\section{Abstract}

6 Quantifying the distribution and size of home ranges is critical for understanding animal spatial

7 dynamics. This is particularly important for large carnivores that reside in fragmented

8 landscapes. Most studies that estimate home range consider only a bivariate frequency

9 distribution represented by a two-dimensional planimetric surface. The underlying assumption of

10 these approaches is that the animals inhabit landscapes that are completely flat. Of course, this is

11 rarely the case. Here we investigated the influence of vertical relief and three-dimensional

12 landscape features on the home range patterns of a high density carnivore. Via GPS telemetry-

13 tracking of a population of Persian leopards (Panthera pardus saxicolor, $\mathrm{n}=6$ ), and globally-

14 available digital elevation models (DEMs), we calculated the surface area of home ranges in

15 comparison to traditional planimetric estimates. We also investigated predation patterns of

16 leopards across elevation gradients using GPS location data and kill site analysis. The

17 topographic measurements exceeded planimetric estimates by up to $38 \%$ which suggests that

18 planimetric modeling underestimates home range size, particularly when animals inhabit variable

19 terrain. We also observed that resident leopards exhibit significant altitudinal partitioning of

20 predation, suggesting that leopards that have overlapping home ranges may still utilize exclusive

21 hunting territories. We discuss the ways in which planimetric approaches may be

22 underestimating aspects of animal ranging behavior and ecology. We conclude that topography

23 should be considered, not as an ancillary metric, but as an important aspect of home range

24 calculation. Our approach can enhance understanding of spatial requirements, population 
25 density, intra-guild sympatric competition and conflict management of large felids inhabiting

26 rugged landscapes.

27

28 Keywords: auto-correlated Kernel density estimation; elevation gradient; intraspecific

29 competition; planimetric; predation pattern; topography.

\section{Introduction}

Animal space use and the scales at which they range, establish home ranges, and use

33 habitat within the home ranges is fundamental to their ecology (Johnson 1980, Börger et al.

34 2008). A home range is integral to animals' cognitive maps of their environment (Powell and

35 Mitchell 2012). At a more basic level, a home range is comprised of abiotic and biotic conditions

36 that have implications for animal survival and reproduction (Burt 1943). How an animal uses

37 different parts of that range can be quantified as a utilization distribution (UD) based on the

38 relative probability of using different parts of the range (Worton 1989) which can provide better

39 understanding of complex habitat dynamics (Worton 1987). Modern statistical modeling of home

40 ranges facilitates the exploration of processes which determine the characteristics of the

41 emergent UDs (Börger et al. 2008).

42 The spatiotemporal availability of resources is an important factor that affects animal

43 movement and the configuration and size of UDs. Ranging patterns of large carnivores are

44 influenced by intrinsic factors such as age and sex (Johansson et al. 2016, Morato et al. 2016) or

45 extrinsic factors including resource availability (Allen et al. 2016, Johansson et al. 2018),

46 topographic features (Powell and Mitchell 1998) and population density (Cooper 1978, Benson

47 et al. 2006). 
The conventional method for quantifying ranging patterns is calculating UDs as a

49 bivariate frequency distribution represented by a two-dimensional $(X, Y)$ planimetric surface

50 (Van Winkle 1975). Kernel-based nonparametric probabilistic models are widely popular for

51 estimating home range size using relocation data (Worton 1989, Laver and Kelly 2008, Fleming

52 and Calabrese 2017). Home ranges and core areas for large carnivores are often quantified

53 without tangibly incorporating topography, notwithstanding being in areas with high orography

54 (Johansson et al. 2016, 2018, Farhadinia et al. 2018a).

55 Topography, however, is often an important influence on animal ecology. Vertical relief

56 can affect the animal's perception of habitat, food resources, access to mates, refugia, or escape

57 from predators (Powell and Mitchell 1998, Stankowich and Coss 2006, Montgomery et al. 2012).

58 Incorporating elevation can provide better insight into coexistence of sympatric large carnivores

59 (Husseman et al. 2003, Li et al. 2018). For example, elevation gradient can have differentiating

60 effect both on selection of habitat and location of kill sites within a guild of sympatric carnivores

61 (Husseman et al. 2003, May et al. 2008). In environments that exhibit strong seasonality, plant

62 phenology can drive vertical migration of herbivores and consequently, synchronize predator

63 movement (Mysterud 2013, Spitz et al. 2018). Ignoring this factor can lead to misinterpretation

64 of animals' ecological needs, particularly in mountainous landscapes. It also can affect our

65 comparative understanding on density,

Home range estimates based on planimetric area, without accounting for the true surface

67 area traversed by an animal, may underestimate home ranges and overestimate population

68 densities in montane regions (Monterroso et al. 2013). Despite the recognized importance of

69 including topography in the estimation of home-range sizes (Powell and Mitchell 1998), few

70 studies endeavored to do so in terrestrial home range estimations (Campbell et al. 2004, 
71 Monterroso et al. 2013, Powell et al. 2017). Importantly, ignoring the true surface can result in

72 the overestimation of population parameters, inducing researchers to consider that the status of

73 population is better than it really is (Monterroso et al. 2013). To our knowledge, no study has

74 included the complexity of topography for terrestrial large carnivore home ranges or core areas.

75 We address this challenge with a GPS telemetry study of the Persian leopard Panthera pardus

76 saxicolor in a remote montane landscape along the Iran-Turkmenistan borderland.

77 Human persecution, mainly in retaliation of killing domestic animals, is a major threat to

78 Persian leopards (Babrgir et al. 2017, Naderi et al. 2018). Their extensive spatial requirements

79 relative to the small size of many reserves means that their persistence often relies on a mosaic of

80 protected and human-altered landscapes (Farhadinia et al. 2018b, Hosseini et al. 2019, Soofi et

81 al. 2019), at least for dispersal or foraging (Sharbafi et al. 2016, Farhadinia et al. 2018a). The

82 unfolding complexities of space use in these highly heterogeneous mountains are likely to inform

83 management plans.

84 We extended our previous planimetric study on ranging patterns (Farhadinia et al.

85 2018a) by incorporating elevation in estimating space use. Using GPS relocation data from a

86 wide-ranging predator living in a rugged mountainous landscape, we modeled the variation

87 between planimetric (two-dimensional) and topographic (three-dimensional) home range and

88 core area. We also explored the association between predation patterns and elevation gradient

89 and how they relate to ecological outcomes at population and intraguild levels. Finally, we

90 discuss how topographic analysis can improve our understanding of the ecology of the species

91 and the corresponding implications for conservation. 


\section{Material and Methods}

\section{Ethics statement}

94 The study was conducted in Tandoureh National Park (TNP), Iran. The Iranian

95 Department of Environment reviewed all sampling, trapping, and handling procedures and

96 approved permits for the work conducted (93/16270). The trapping and handling protocols were

97 also approved by the University of Oxford's Ethical Review Committee (BMS-ERC-160614).

98 Study area

99 We studied leopards in TNP, a $355 \mathrm{~km}^{2}$ national park in north-eastern Iran approximately

$10020 \mathrm{~km}$ from the Turkmenistan border. Protected since 1968, TNP is characterized by a dry

101 climate with average annual rainfall of between 250 and $300 \mathrm{~mm}$. The vegetation of the area's

102 mountains are dominated by wormwood Artemisia sp. and scattered juniper trees Juniperus sp.

103 Elevation ranges from 1,000 to $2,600 \mathrm{~m}$.

104 The primary occupation of local human communities is sheep and goat herding in

105 villages with populations typically ranging between 30 to 400 households. There are no human

106 settlements inside the park. Leopards in TNP mainly prey on urial (Ovis orientalis), bezoar goat

107 (Capra aegagrus), and wild pig (Sus scrofa). Apart from wild pigs, which are occasionally found

108 in multi-use areas outside of the national park, other wild medium-sized prey are available only

109 inside the park. In contrast, domestic animals are found exclusively outside the park (Farhadinia

110 et al. 2018c).

\section{Leopard collaring and monitoring}

112 We collared six leopards (5 males and 1 female; Table 1) using Aldrich foot-snare traps

113 (see Farhadinia et al. 2017a for details on capturing and immobilization procedures). Age 
114 determination was based on dental features (see Farhadinia et al. 2017a for more details). We

115 used GPS collars with Iridium download capabilities (LOTEK Engineering Ltd., Newmarket,

116 ON, Canada), each equipping with a drop-off buckle activated by a timer set to 52 weeks since

117 deployment.

118 To increase fix success rates, fixes were taken hourly during the last week of each month

119 whereas for the rest of month, fixes were recorded every 3 hours. This programming schedule

120 was adopted to facilitate the identification of spatially aggregated GPS points, or clusters

121 indicative of kill sites (Knopff et al. 2009) while securing the battery life until the end of collar

122 activity. Bjørneraas et al. (2010) recommended that to analyse animal movement and behaviour,

123 fixes obtained immediately after collaring should be excluded because the animal may not

124 behave normally. Therefore, we omitted all data from the first 4 days post-collaring, associated

125 with the earliest known kill made by the leopards after collaring (M1) (Farhadinia et al. 2018c).

126 We investigated potential kill sites defined as clusters of $\geq 2$ locations within $100 \mathrm{~m}$ of

127 each other and separated by less than 12 hour (Knopff et al. 2009, see Farhadinia et al. 2018c for

128 more details). We only included "medium" prey ( $\geq 15 \mathrm{~kg})$ such as urial, bezoar goat, wild pig,

129 domestic sheep Ovis aries and domestic dog Canis familiaris into our elevation analysis.

130

131

132

133

134

135

136

\section{Home range and core area calculation}

In our previous study (Farhadinia et al. 2018a), we did not detect any erroneous fixes or spikes in movement in our GPS relocation data, based on identification of locations arising from unrealistic movement patterns, using a script developed by Bjørneraas et al. (2010).

We developed UDs to map home ranges of the leopards using an auto-correlated kernel density estimator (AKDE). Relocation data are inherently auto-correlated (i.e. an individual's position, velocity, or acceleration are statistically correlated with the same measurements in the 
137 past and future unless inter-location intervals are long enough) (Fleming et al. 2015, Fleming and

138 Calabrese 2017). The AKDE is a continuous-time method that accounts for auto-correlated

139 bivariate Gaussian density estimation for relocation data (Fleming et al. 2015).

140 We investigated the autocorrelation structure of the relocation data for each leopard using

141 an empirical variogram. It is defined as the estimated semi-variance in positions as a function of

142 the time lag separating observations. Upward curvature at zero to short time lags indicates

143 velocity autocorrelation while the long-lag behavior of the variogram illustrates space use

144 (Fleming and Calabrese 2017). Thus, range residents are expected to reach an asymptote on a

145 timescale that roughly corresponds to the home-range crossing time (Calabrese et al. 2016).

146 We implemented two movement models, Ornstein-Uhlenbeck (OU) and Ornstein-

147 Uhlenbeck Foraging (OUF), in package 'ctmm' version 0.4.0 (Calabrese et al. 2016). They both

148 accommodate auto-correlated data to quantify home range size. Accordingly, the OU process

149 combines a random search model without space use constraint (Brownian motion) with a

150 tendency to remain in a particular home range. Alternatively, the OUF process features both

151 velocity autocorrelation and restricted space use (Calabrese et al. 2016, Fleming and Calabrese

152 2017). Initial values of the model parameters were obtained from variograms overlaid with a

153 continuous-time movement model. For each leopard, movement models were fitted using

154 maximum likelihood estimation to the data. We selected the best movement model using AICc

155 weight to estimate home range for each leopard, defined as area within $95 \%$ \%D isopleths of

156 AKDE estimates.

157 We also estimated core areas of space use for each leopard using an individual-based

158 quantitative approach (Vander Wal and Rodgers 2012). Thus, the UDs were plotted against

159 isopleths to identify the value at which the proportional home range area begins to increase at a 
160 greater rate than the probability of use $($ slope $=1)$. The value of the corresponding isopleth

161 determines the boundary of the core area (Vander Wal and Rodgers 2012).

162

163

164

165

166

167

168

169

170

171

172

173

174

175

176

177 spatial polygons generated by our UD estimation for both home range and core area of all

178 leopards. These polygons were the result of mathematical formula, and therefore have smooth 179 edges that do not perfectly match the boundaries of a raster grid. Grid cells were included in the 180 masked raster whose centroid lay within the polygon. 
We derived elevation values for each kill site, recorded for each GPS fix by the collar.

183 We developed a generalized linear model to relate elevation of kills to prey type. Another

184 generalized linear model was constructed to explore elevation variability in kill sites for each

185 leopard. For the latter analysis, we omitted non-resident individuals for which predation can

186 occur less-predictably in the landscape. For example, most of scavenging clusters belonged to

187 non-resident leopards in TNP (Farhadinia et al. 2018c). For one individual (B1/Borzou), we

188 truncated the first 5.5 months for which he showed residency behavior (see Home range and core

189 area calculation for details on differentiating between resident and non-resident individuals). All

190 means are reported with standard errors. We conducted all analyses in $R$ (R Development Core

191 Team 2013).

\section{Data deposition}

194 All locational data are publicly available on Movebank:

195 https://www.movebank.org/panel_embedded_movebank_webapp. Project: Persian leopard

196 Tandoureh Iran (accession number 270329098). All the data are available for download as .csv

197 file.

\section{Results}

200 We monitored six leopards for between 54 and 368 days per individual (Table 1). In total, 201 they represented a study period of 56.7 leopard months, monitored between September 2014 and 202 May 2017. We obtained a total of 22,226 GPS locations for 1,702 leopard-days (283.7 \pm SE 50.8 203 days/leopard) with a high overall fix rate (mean 85.0\% \pm SE 7.6). 
Three adult males (M2/Bardia, M3/Borna and M4/Tandoureh) exhibited constrained

205 space use as resident individuals, based on a clear asymptote in variograms (Fig. 1 and

206 Supplementary material Appendix 1, Fig. 1). In contrast, both young leopards (F5/Iran and

207 M6/Kaveh) lacked asymptotes, showing a non-residency pattern. M1/Borzou (an old male)

208 showed a mixed ranging pattern. Following 5.5 months of residency behavior inside the national

209 park, he started his excursions outside the park along the borderland's communities with regular

210 returns to the national park which was associated with an increase in his semi-variance (Fig. 1

211 and Supplementary material Appendix 1, Fig. 1). Although M4/Tandoureh was collared when

212 other males' collars were dropped off, photographic detections by means of camera traps or

213 tourist encounters suggested that he was a resident male in the same area before collaring.

214 Therefore, we used his relocation data to analyze inter-individual altitudinal differences in 215 predation patterns.

216 The AKDE estimates were obtained for all six leopards equally shared between OU and

217 OUF as best performing models based on their AICc weight (Table 1). Mean planimetric AKDE

218 home range was calculated to be $103.4 \pm \mathrm{SE} 51.8 \mathrm{~km}^{2}$ for resident males while it increased to

$219133.2 \pm$ SE $65.5 \mathrm{~km}^{2}$ when topographically analyzed. Similarly, mean core area was calculated to

220 be $32.4 \pm \mathrm{SE} 12.7 \mathrm{~km}^{2}$ planimetrically with a mean surface area of $42.3 \pm \mathrm{SE} 16.3 \mathrm{~km}^{2}$ (Table 1).

221 The mean isopleth for core areas were the $62 \%$ to $67 \%$ isopleths of the utility distribution.

222 Calculation of surface area was increased by a median of $27.8 \%$ for all collared leopards

223 when topography was also incorporated. Specifically, home range size and core area estimates

224 increased by $28.4 \pm$ SE $1.2 \%$ and $29.6 \pm$ SE $1.8 \%$, respectively (Table 1$)$. The increase in spatial

225 parameters for all three resident leopards was above the median line, whereas it was slightly 
226 lower for non-residents (Fig. 2). At individual level, the leopards' ranges showed a variable

227 factor of increase between $26.0 \%$ and $38.4 \%$ (Fig. 2).

228 In total, we detected 139 kill sites where leopards preyed $(n=130)$ or scavenged $(n=9)$

229 on 10 different prey species. The majority (93.1\%) of these prey were medium sized. The

230 elevation at which these predations took place varied between leopards $\left(\mathrm{F}_{3,91}=28.32, P<0.005\right.$;

231 Fig. 3). Also, various medium sized prey were killed at different elevations $\left(\mathrm{F}_{4,120}=12.16, P<\right.$

232 0.005), with two clear groups identifiable: lowland prey including sheep, domestic dog and wild

233 pig (ca. 1100 meter a.s.1), and highland prey such as urial and bezoar goat (ca. 1500 meter a.s.l;

234 Fig. 4).

235

236 Discussion

237 We observed that elevation is an important component in representing the home range

238 behavior of a large carnivore. We found that failing to consider elevation in home range

239 estimation led to a $38 \%$ bias in home range and core area estimation in our mountainous study

240 area. We would not expect this bias to be consistent across space. Rather, it should dynamically

241 vary given the landscape and the life history characteristics of leopards. In this case, resident

242 male leopards showed elevation-dependent predation behavior, while non-resident leopards had

243 high elevation variability in ranging patterns.

244 Topographic versus planimetric spatial analysis

245 The use of topographic home range estimation facilitated examination of the importance

246 of elevation in studies of leopard space use. Our results confirmed that the incorporation of

247 elevation into home range modeling can significantly increase the range size. Conversely, our

248 study demonstrates the tendency for planimetric quantification of terrestrial home range to 
249 underestimate the surface size used in montane areas; however, planimetric home range

250 techniques are likely valid in areas with less topographic relief (Walter et al. 2013). Simulation

251 studies suggest that planimetric estimates in areas with an average slope of $16^{\circ}$ or more will

252 incur in errors $\geq 5 \%$ (Monterroso et al. 2013). Regardless of the analysis method, our home

253 range estimates for Persian leopards are larger than those reported in previous Asian leopard

254 ranging studies (Odden and Wegge 2005, Simcharoen et al. 2008).

255 The method we used here for surface area calculation, first developed by Jenness (2004),

256 does not require a great amount of additional effort or computational efficiency, and we believe

257 it provides a more realistic estimate of space use. We therefore encourage ecologists to

258 incorporate elevation and topography into their movement ecology analysis in mountainous

259 environments to obtain a more complete understanding of species spatial behavior.

260 Ecological outcomes along elevation gradient

261 Large felids in rugged landscapes show moderate home range overlap between

262 conspecifics (Farhadinia et al. 2018a, Johansson et al. 2018). Several plausible mechanisms

263 could explain the coexistence of multiple individuals. First, although long-term home range

264 overlap can be relatively high, short- term home overlaps can be much smaller (Johansson et al.

265 2018). Second, despite high degrees of spatial overlap, predators can have exclusive hunting

266 areas, which was seen in TNP (Farhadinia et al. 2018a). Finally, as shown in this study, different

267 predators, despite sharing large parts of their home range, confine their hunting areas to

268 individual-specific elevations. All these three mechanisms facilitate intraspecific competitive

269 exclusion which is widely reported in territorial carnivores (Steyn and Funston 2009, Lopez-Bao

270 et al. 2011, Broekhuis et al. 2018). 
Leopards showed elevation predation patterns, with bezoar goat and urial as highland

272 prey whereas lowland prey include sheep, domestic dog and wild pig. This pattern can have two

273 implications. First, in strongly seasonal environments, plant phenology can drive elevation

274 migration of herbivores and consequently, synchronizing predator movement (Mysterud 2013,

275 Bleyhl et al. 2019). Thus, integrating elevation gradients of resources for prey and predators in

276 rugged landscapes can improve ecological interpretation and management plans. Although our

277 sample size was not large enough to evaluate the effect of season, future research with larger

278 sample sizes can test it on prey-predator interaction across elevation gradients. Second, domestic

279 dog and sheep were mainly killed in lowland areas, suggesting that conflict with rural people can

280 be a problem happening at lower levels of an elevation gradient. The practices of prey recovery

281 through establishing law enforcement as well as minimizing wild ungulate competition with

282 livestock and associated displacement from optimal habitats are widely encouraged (Babrgir et

283 al. 2017, Karimov et al. 2018, Bleyhl et al. 2019, Soofi et al. 2019). Our findings highlight the

284 importance of considering elevation in conflict resolution plans through elevation zoning efforts.

285 Elevation gradients and vertical relief can shape fine-scale processes impacting

286 mammalian carnivore communities. Two key axes of co-occurrence include spatial and dietary

287 overlap in a guild of large carnivores (May et al. 2008, Davis et al. 2018). For example, although

288 wolves Canis lupus are rarely detected in the national park's rugged highlands; they commonly

289 attack livestock in lowland pastures outside the park (Farhadinia et al. 2017b). In terms of dietary

290 requirement, wolves primarily prey on lowland prey species such as plain-dwelling ungulates,

291 livestock and anthropogenic resources (Hosseini-Zavarei et al. 2013, Chetri et al. 2017). In

292 contrast, leopards' main prey are ungulates inhabiting higher elevations (Sharbafi et al. 2016,

293 Farhadinia et al. 2018c, Soofi et al. 2019). Thus, differentiated patterns in human-carnivore 
294 conflict can occur, for example in terms of livestock type raided (Babrgir et al. 2017, Soofi et al. 295 2019) or seasonality (Hosseini-Zavarei et al. 2013, Chetri et al. 2017). Habitat segregation across 296 elevation gradients and prey separation help to avoid intraguild interactions at the site-specific

297 levels in mountainous environments with strong seasonal variation.

299 understanding the variability in their density and what affects it is of continuing interest for 300 ecologists (Hearn et al. 2017, Suryawanshi et al. 2019). Although great variation in density 301 estimates of leopards is seen across their global range (Vitkalova et al. 2018, Balme et al. 2019), 302 they can reach exceptionally high densities in montane environments (Grey et al. 2013, Williams 303 et al. 2017). Our findings suggest that without incorporating elevation and topographic relief in 304 surface estimation, density measures can potentially be overestimated in mountainous 305 landscapes. Therefore, comparing density estimates across different ecosystems types with 306 varied levels of topographic ruggedness and elevation variability needs to be done cautiously.

\section{Synthesis and conclusion}

309 shaping ranging and foraging behaviour of a large carnivore in a rugged landscape. Future

310 research might usefully explore inter-sexual differences space use and predation patterns of

311 leopards across elevation gradients. Resident females generally have smaller home ranges

312 (Simcharoen et al. 2008, Johansson et al. 2018) and consequently are expected to show less 313 variability across the elevation gradients.

314 Mountains, covering $25 \%$ of the global land surface area, are regularly a focus of 315 international efforts to conserve a wide range of ecosystem services and biodiversity (Körner 316 2007). Mountainous areas presently act as refugia for large Asian felids in many range states, 
317 often because such habitat presents obstacles to human inhabitation. However, they are under

318 increasing pressure from multiple threats whose function and effect can vary along the elevation

319 gradients (Jacobson et al. 2016, Li et al. 2018, Penjor et al. 2018, Vitkalova et al. 2018,

320 Farhadinia et al. 2019, Suryawanshi et al. 2019). Therefore, understanding the dynamic

321 interaction between species requirement and resource availability along the elevation gradient

322 can effectively inform protection plans.

323

324

325 Acknowledgements - We sincerely thank the Iranian Department of Environment for

326 administrative support and provision of necessary permissions. The SRTM GDEM data product

327 used in the preparation of our results were retrieved from the online Data Pool, courtesy of

328 NASA's Earth Observing System Data and Information System (EOSDIS)

329 (https://earthexplorer.usgs.gov), USGS/Earth Resources Observation and Science (EROS)

330 Center, Sioux Falls, South Dakota, USA. I. Memarian, B. Ekrami and J. Kaandorp assisted in

331 leopard capturing and anesthesia. Ch. Fleming, E. Vander Wal and J. Thompson provided

332 constructive discussion for data analysis and interpretation. We are very grateful to the field

333 crew, particularly P. Behnoud, P. Moghadas, A. Shahrdari, K. Baradarani, Z. Salahshour, S.

334 Firouzi, A. Daneshvar, B. Jalali, and Gh. Safdari for their field assistance. 
336 Funding - This study was funded by the People's Trust for Endangered Species (PTES),

337 Zoologische Gesellschaft für Arten- und Populationsschutz (ZGAP), Iranian Cheetah Society,

338 Quagga Conservation Fund, IdeaWild and Association Francaise des Parcs Zoologiques

339 (AFdPZ).

340

341 Author contributions - MSF, DRH and RAM conceived the ideas and designed methodology;

342 MSF and KH collected the data; MSF, DRH, RAM and PJJ analysed the data; MSF, DRH and

343 RAM led the writing of the manuscript. All authors contributed critically to the drafts and gave

344 final approval for publication.

345

346 Conflict of interest - The authors have no conflicts of interest to declare.

347

348 Permit(s) - The study was conducted in Tandoureh National Park (TNP), Iran. The Iranian

349 Department of Environment reviewed all sampling, trapping, and handling procedures and

350 approved permits for the work conducted (93/16270). The trapping and handling protocols were

351 also approved by the University of Oxford's Ethical Review Committee (BMS-ERC-160614).

354 References

355 Allen, A. M. et al. 2016. Scaling up movements: from individual space use to population 356 patterns. - Ecosphere in press.

357 Babrgir, S. et al. 2017. Socio-economic consequences of cattle predation by the Endangered 358 Persian leopard Panthera pardus saxicolor in a Caucasian conflict hotspot, northern Iran. $359 \quad$ Oryx 51: 124-130. 
360 Balme, G. et al. 2019. Big cats at large: Density, structure, and spatio-temporal patterns of a 361 leopard population free of anthropogenic mortality. - Popul. Ecol. in press.

362 Benson, J. F. et al. 2006. Regulation of space use in a solitary felid: population density or prey 363 availability. - Anim. Behav. 71: 685-693.

364 Bjørneraas, K. K. et al. 2010. Screening Global Positioning System Location Data for Errors 365 Using Animal Movement Characteristics. - J. Wildl. Manage. 74: 1361-1366.

Bleyhl, B. et al. 2019. Assessing niche overlap between domestic and threatened wild sheep to 367 identify conservation priority areas. - Divers. Distrib. 25: 129-141.

368 Börger, L. et al. 2008. Are there general mechanisms of animal home range behaviour? A review 369 and prospects for future research. - Ecol. Lett. 11: 637-650.

370 Broekhuis, F. et al. 2018. Feeding ecology of cheetahs in the Maasai Mara, Kenya and the 371 potential for intra-and interspecific competition. - J. Zool. 304: 65-72.

372 Burt, W. H. 1943. Territoriality and home range concepts as applied to mammals. - J. Mammal. $373 \quad 24: 346-352$.

374 Calabrese, J. M. et al. 2016. ctmm: an r package for analyzing animal relocation data as a 375 continuous-time stochastic process. - Methods Ecol. Evol. 7: 1124-1132.

376 Campbell, T. A. et al. 2004. Topographic home ranges of white-tailed deer in the central 377 Appalachians. - Southeast. Nat. 3: 645-653.

378 Chetri, M. et al. 2017. Snow Leopard and Himalayan Wolf: Food Habits and Prey Selection in 379 the Central Himalayas, Nepal. - PLoS One 12: e0170549.

380 Cooper, W. E. 1978. Home range size and population dynamic. - J. Theor. Biol. 75: 327-337.

381 Davis, C. L. et al. 2018. Ecological correlates of the spatial co-occurrence of sympatric 382 mammalian carnivores worldwide. - Ecol. Lett. 21: 1401-1412. 
383 Farhadinia, M. S. et al. 2017a. GPS collars reveal transboundary movements by Persian leopards 384 in Iran. - Cat News 65: 28-30.

385 Farhadinia, M. S. et al. 2017b. Wolves can suppress goodwill for leopards: Patterns of human386 predator coexistence in northeastern Iran. - Biol. Conserv. 213: 210-217.

387 Farhadinia, M. S. et al. 2018a. Anchoring and adjusting amidst humans: Ranging behavior of 388 Persian leopards along the Iran-Turkmenistan borderland. - PLoS One 13: e0196602.

389 Farhadinia, M. S. et al. 2018b. Citizen science data facilitate monitoring of rare large carnivores 390 in remote montane landscapes. - Ecol. Indic. 94: 283-291.

391 Farhadinia, M. S. et al. 2018c. Persian leopard predation patterns and kill rates in the Iran392 Turkmenistan borderland. - J. Mammal. 99: 713-723.

393 Farhadinia, M. S. et al. 2019. Belt and Road Initiative may create new supplies for illegal 394 wildlife trade in large carnivores. - Nat. Ecol. Evol.: 1-2.

395 Fleming, C. H. and Calabrese, J. M. 2017. A new kernel density estimator for accurate home396 range and species-range area estimation. - Methods Ecol. Evol. 8: 571-579.

397 Fleming, C. H. et al. 2015. Rigorous home range estimation with movement data: a new 398 autocorrelated kernel density estimator. - Ecology 96: 1182-1188.

399 Grey, J. N. C. et al. 2013. Evidence of a high density population of harvested leopards in a 400 montane environment. - PLoS One 8: e82832.

401 Hearn, A. J. et al. 2017. Responses of Sunda clouded leopard Neofelis diardi population density 402 to anthropogenic disturbance: refining estimates of its conservation status in Sabah. - Oryx: $403 \quad 1-11$.

404 Hijmans, R. J. et al. 2017. Package 'geosphere.' - Retrieved from in press.

405 Hosseini-Zavarei, F. et al. 2013. Predation by grey wolf on wild ungulates and livestock in 
central Iran. - J. Zool. in press.

407 Hosseini, M. et al. 2019. Landscape connectivity for mammalian megafauna along the Iran-

408 Turkmenistan-Afghanistan borderland. - J. Nat. Conserv.: 125735.

409 Husseman, J. S. et al. 2003. Assessing differential prey selection patterns between two sympatric $410 \quad$ large carnivores. - Oikos 101: 591-601.

411 Jacobson, A. P. et al. 2016. Leopard (Panthera pardus) status, distribution, and the research 412 efforts across its range. - PeerJ 4: e1974.

413 Jenness, J. S. 2004. Calculating landscape surface area from digital elevation models. - Wildl.

$414 \quad$ Soc. Bull. 32: 829-840.

415 Johansson, Ö. et al. 2016. Land sharing is essential for snow leopard conservation. - Biol.

416 Conserv. 203: 1-7.

417 Johansson, Ö. et al. 2018. Sex-specific seasonal variation in puma and snow leopard home range $418 \quad$ utilization. - Ecosphere 9: e02371.

419 Johnson, D. H. 1980. The comparison of usage and availability measurements for evaluating $420 \quad$ resource preference. - Ecology 61: 65-71.

421 Karimov, K. et al. 2018. Responses of snow leopards, wolves and wild ungulates to livestock 422 grazing in the Zorkul Strictly Protected Area, Tajikistan. - PLoS One 13: e0208329.

423 Knopff, K. H. et al. 2009. Evaluating global positioning system telemetry techniques for 424 estimating cougar predation patterns. - J. Wildl. Manage. 73: 586-597.

425 Körner, C. 2007. The use of 'altitude' in ecological research. - Trends Ecol. Evol. 22: 569-574.

426 Laver, P. N. and Kelly, M. J. 2008. A Critical Review of Home Range Studies. - J. Wildl.

$427 \quad$ Manage. 72: 290-298.

$428 \mathrm{Li}, \mathrm{Z}$. et al. 2018. Coexistence of two sympatric flagship carnivores in the human-dominated 
forest landscapes of Northeast Asia. - Landsc. Ecol. in press.

430

431

432

433

434 Monterroso, P. et al. 2013. Estimating home-range size: when to include a third dimension? 435
Lopez-Bao, J. V et al. 2011. Intraspecific interference influences the use of prey hotspots. Oikos 120: 1489-1496.

May, R. et al. 2008. Habitat differentiation within the large-carnivore community of Norway's multiple-use landscapes. - J. Appl. Ecol. 45: 1382-1391.
Montgomery, R. A. et al. 2012. Importance of visibility when evaluating animal response to roads. - Wildlife Biol. 18: 393-406.

Morato, R. G. et al. 2016. Space use and movement of a neotropical top predator: the endangered jaguar. - PLoS One 11: e0168176.

Mysterud, A. 2013. Ungulate migration, plant phenology, and large carnivores: The times they are a-changin'. - Ecology 94: 1257-1261.

Naderi, M. et al. 2018. Persian leopard's (Panthera pardus saxicolor) unnatural mortality factors analysis in Iran. - PLoS One 13: e0195387.

Odden, M. and Wegge, P. 2005. Spacing and activity patterns of leopards Panthera pardus in the Royal Bardia National Park, Nepal. - Wildlife Biol. 11: 145-152.

Pebesma, E. and Bivand, R. 2005. sp: classes and methods for spatial data. R package version $0.7-12$. in press.

Penjor, U. et al. 2018. Identifying important conservation areas for the clouded leopard Neofelis nebulosa in a mountainous landscape: Inference from spatial modeling techniques. - Ecol. Evol. 8: 4278-4291.

Powell, R. A. and Mitchell, M. S. 1998. Topographical constraints and home range quality. - 

Ecography (Cop.). 21: 337-341.

453 Powell, R. A. and Mitchell, M. S. 2012. What is a home range? - J. Mammal. 93: 948-958.

454 Powell, R. A. et al. 2017. Stink or swim-techniques to meet the challenges for the study and 455 conservation of small critters that hide, swim or climb and may otherwise make themselves 456 unpleasant. - Biol. Conserv. wild musteloids in press.

457 R Development Core Team 2013. R: A language and environment for statistical computing. in $458 \quad$ press.

459 Sharbafi, E. et al. 2016. Prey of the Persian Leopard (Panthera pardus saxicolor) in a mixed 460 forest-steppe landscape in northeastern Iran (Mammalia: Felidae). - Zool. Middle East 62: 1-8.

462 Simcharoen, S. et al. 2008. Home range size and daytime habitat selection of leopards in Huai 463 Khaeng Wildlife Sanctuary, Thailand. - Biol. Conserv. 141: 2242-2250.

464 Soofi, M. et al. 2019. Assessing the relationship between illegal hunting of ungulates, wild prey 465 occurrence and livestock depredation rate by large carnivores. - J. Appl. Ecol. 56: 365-374.

Spitz, D. B. et al. 2018. How plastic is migratory behavior? Quantifying elevational movement in a partially migratory alpine ungulate, the Sierra Nevada bighorn sheep (Ovis canadensis sierrae). - Can. J. Zool. 96: 1385-1394.

Stankowich, T. and Coss, R. G. 2006. Effects of risk assessment, predator behavior, and habitat on escape behavior in Columbian black-tailed deer. - Behav. Ecol. 18: 358-367.

473 Suryawanshi, K. R. et al. 2019. Sampling bias in snow leopard population estimation studies. $474 \quad$ Popul. Ecol. in press. 
475 Van Winkle, W. 1975. Comparison of several probabilistic home-range models. - J. Wildl.

$476 \quad$ Manage.: 118-123.

477 Vander Wal, E. and Rodgers, A. R. 2012. An individual-based quantitative approach for

478 delineating core areas of animal space use. - Ecol. Modell. 224: 48-53.

479 Vitkalova, A. V et al. 2018. Transboundary cooperation improves endangered species

480 monitoring and conservation actions: A case study of the global population of Amur

481 leopards. - Conserv. Lett. 11: e12574.

482 Walter, W. D. et al. 2013. Topographic home range of large mammals: is planimetric home 483 range still a viable method? - Prairie Nat. 45: 21.

484 Williams, S. T. et al. 2017. Population dynamics and threats to an apex predator outside 485 protected areas: implications for carnivore management. - R. Soc. Open Sci. 4: 161090.

486 Worton, B. J. 1987. A review of models of home range for animal movement. - Ecol. Modell. 38:

$487 \quad 277-298$.

488 Worton, B. J. 1989. Kernel methods for estimating the utilization distribution in home-range $489 \quad$ studies. - Ecology 70: 164-168.

490

491

492

493

494 
495 Table 1. Movement parameters, home range estimates, and range size differences between planimetric and surface area of GPS

496 collared Persian leopards (Panthera pardus saxicolor) in Tandoureh National Park, northeastern Iran. Home range estimates were

497 calculated using 95\% utilization distributions (UDs). The mean isopleth for core areas were $62 \%$ to $67 \%$ of the UDs. Home ranges for

498 individuals marked with asterisk $\left(^{*}\right)$ were based on an Ornstein-Uhlenbeck (OU) process model, while an Ornstein-Uhlenbeck

499 Foraging (OUF) process model was fitted to the other animals based on their AICc weight. Means are only based on resident

500 individuals (M2/Bardia, M3/Borna and M4/Tandoureh)

\begin{tabular}{|c|c|c|c|c|c|c|c|c|c|c|}
\hline $\begin{array}{l}\text { Leopard } \\
\text { ID/Name }\end{array}$ & Sex/age & $\begin{array}{c}\text { Capture } \\
\text { date }\end{array}$ & Last fix & \# days & $\begin{array}{c}\text { Planimetric } \\
\text { AKDE } \\
95 \%\left(\mathrm{~km}^{2}\right)\end{array}$ & $\begin{array}{c}\text { Planimetric } \\
\text { Core area } \\
\left(\mathrm{km}^{2}\right)\end{array}$ & $\begin{array}{c}\text { Topographic } \\
\text { AKDE } \\
\left(\mathbf{k m}^{2}\right)\end{array}$ & $\begin{array}{c}\text { Topographic } \\
\text { Core area } \\
\left(\mathrm{km}^{2}\right)\end{array}$ & $\begin{array}{c}\text { AKDE 95\% } \\
\text { 3D/2D size } \\
\text { difference } \\
(\%)\end{array}$ & $\begin{array}{c}\text { Core area } \\
\text { 3D/2D size } \\
\text { difference } \\
(\%)\end{array}$ \\
\hline M1/Borzou* & $\mathrm{M} /+10$ & 5.2 .2015 & 4.2 .2016 & 368 & 563.4 & 224.5 & 709.7 & 287.3 & 26.1 & 27.8 \\
\hline M2/Bardia & $\mathrm{M} / 8-10$ & 3.10 .2014 & 30.9 .2015 & 362 & 43.9 & 16.5 & 58.8 & 22.8 & 34.2 & 38.4 \\
\hline M4/Tandoureh & $\mathrm{M} / 7-10$ & 16.8 .2016 & 1.04 .2017 & 228 & 59.8 & 23.1 & 76.9 & 29.5 & 28.7 & 27.8 \\
\hline F5/Iran & $\mathrm{F} / 2-3$ & 6.12 .2015 & 29.1.2016 & 54 & 330.9 & 92.5 & 420.6 & 117.8 & 27.1 & 27.6 \\
\hline M6/Kaveh* & $M / 3-4$ & 4.9 .2015 & 26.8 .2016 & 326 & 2269 & 775.7 & 2856.1 & 973.9 & 26.0 & 26.0 \\
\hline Mean (SE) & & & & $283.7(50.8)$ & $103.4(51.8)$ & $32.4(12.7)$ & $133.2(65.5)$ & $42.3(16.3)$ & & \\
\hline
\end{tabular}




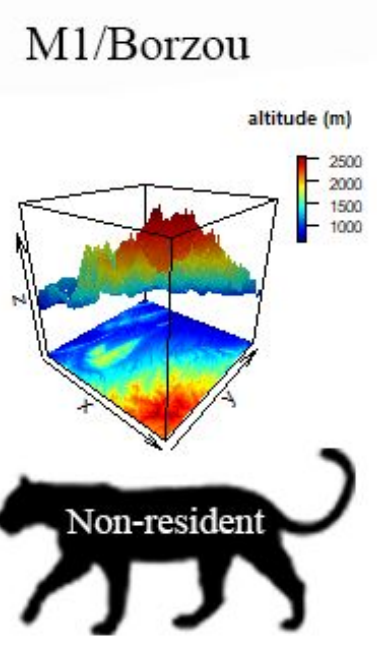

\section{M4/Tandoureh}

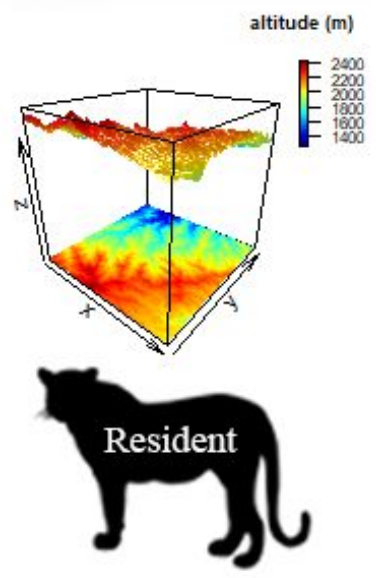

M2/Bardia

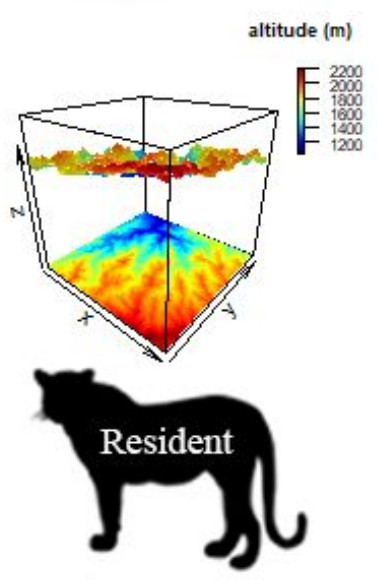

F5/Iran

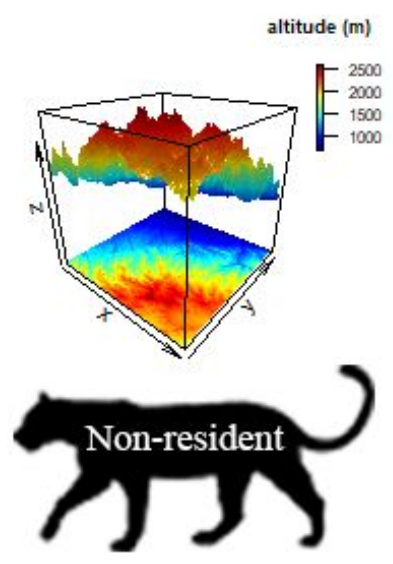

M3/Borna

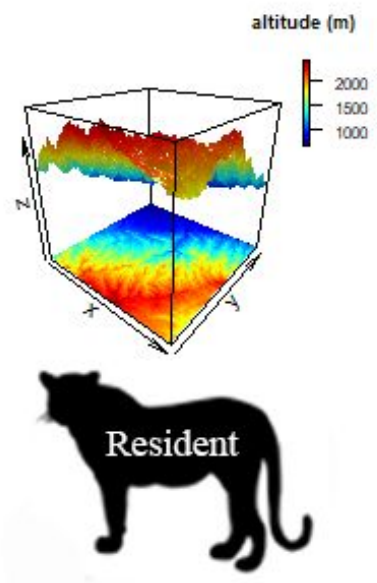

M6/Kaveh

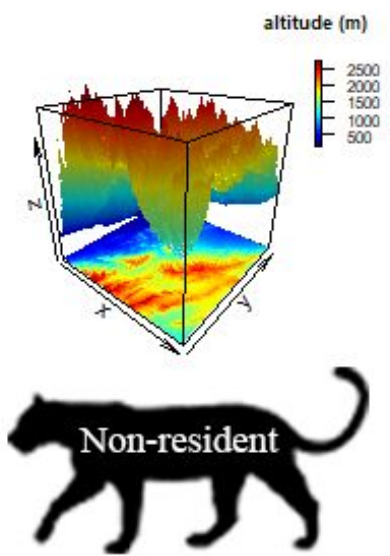

503

504 Figure 1

505 Topographic surface areas for six collared leopards superimposed on masked three-dimensional

506 digital elevation model (DEM) plots. Home ranges were calculated as 95\% utilization

507 distribution (UD) using auto-correlated kernel density estimator (AKDE) of GPS collar data

508 from leopards captured in Tandoureh National Park, northeastern Iran. Individual ranges are not

509 similarly scaled. 


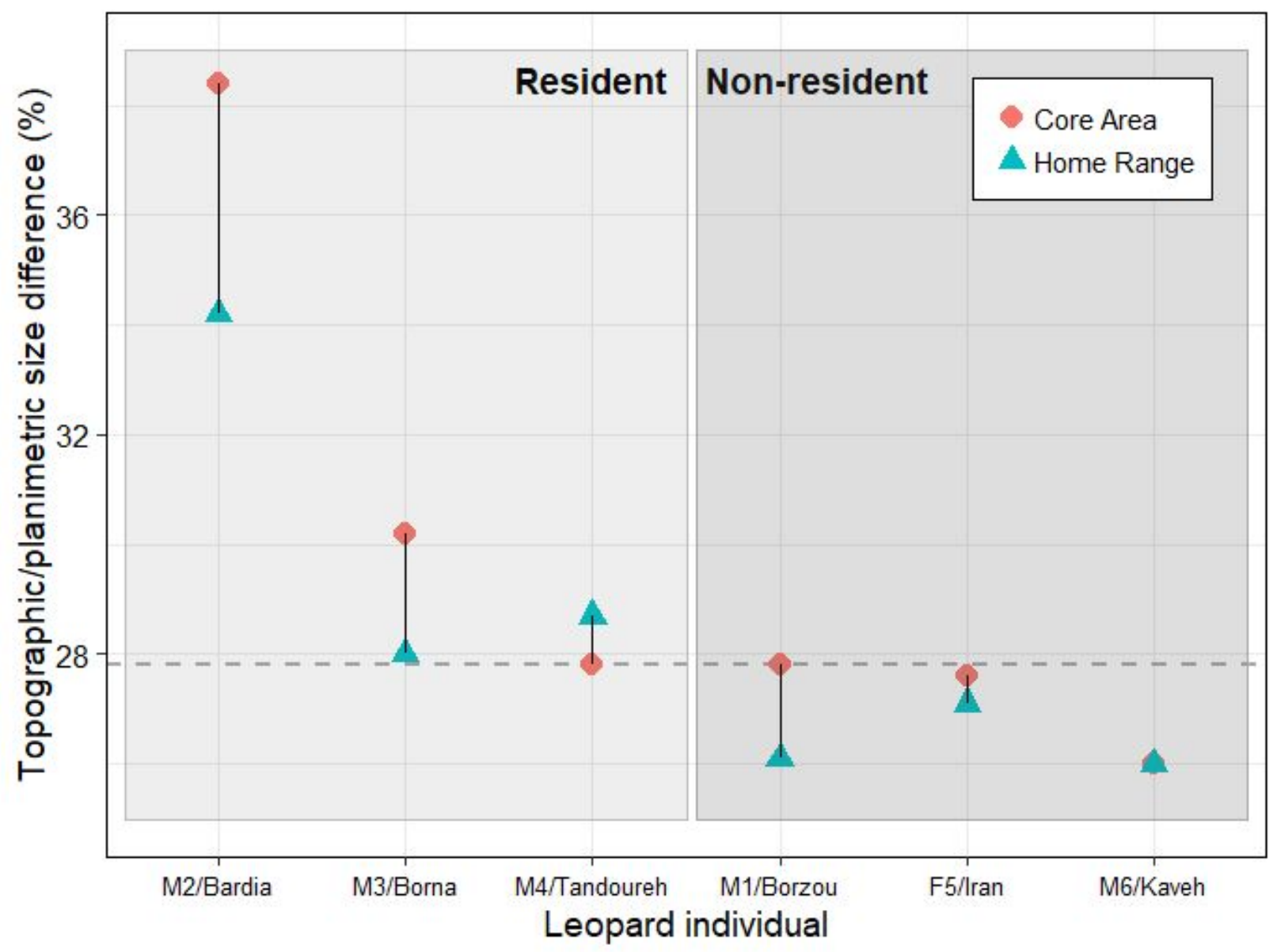

\section{Figure 2}

513 Combined factor of increase when comparing home range and core area of six GPS collared

514 leopards captured in Tandoureh National Park, northeastern Iran. The dashed grey line represents 515 median of topographic/planimetric size difference ratio. 


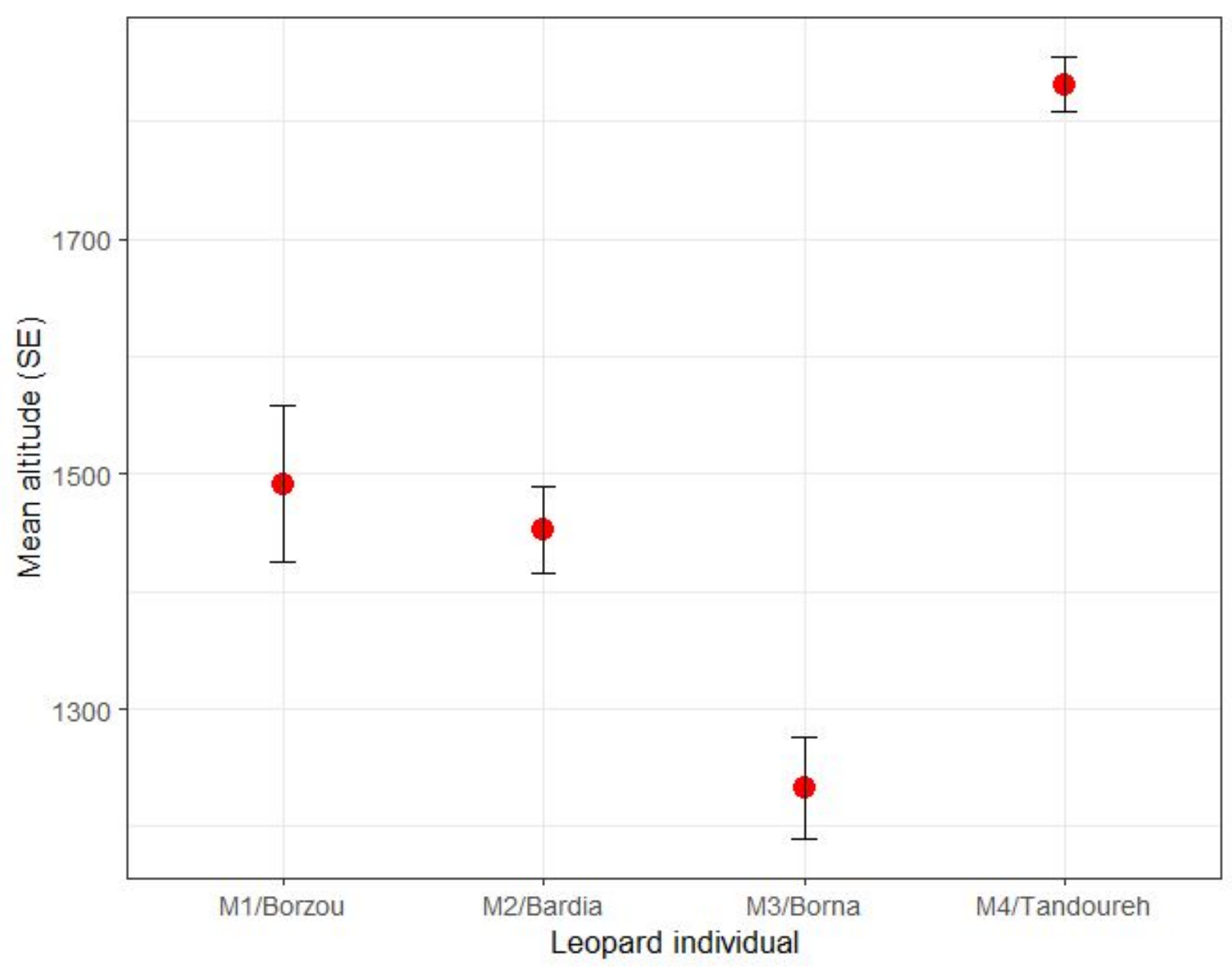

\section{$519 \quad$ Figure 3}

520 Inter-individual altitudinal differences in predation patterns of four adult Persian leopards in

521 Tandoureh National Park. Predation events represent only kills made by resident leopards inside

522 the national park, because all animals did not make kills outside the national park where most

523 prey are lowland prey such as sheep, dog or wild pig. B1/Borzou's first 5.5 months was also

524 included for which he showed residency behavior. Error bars represent standard errors. 


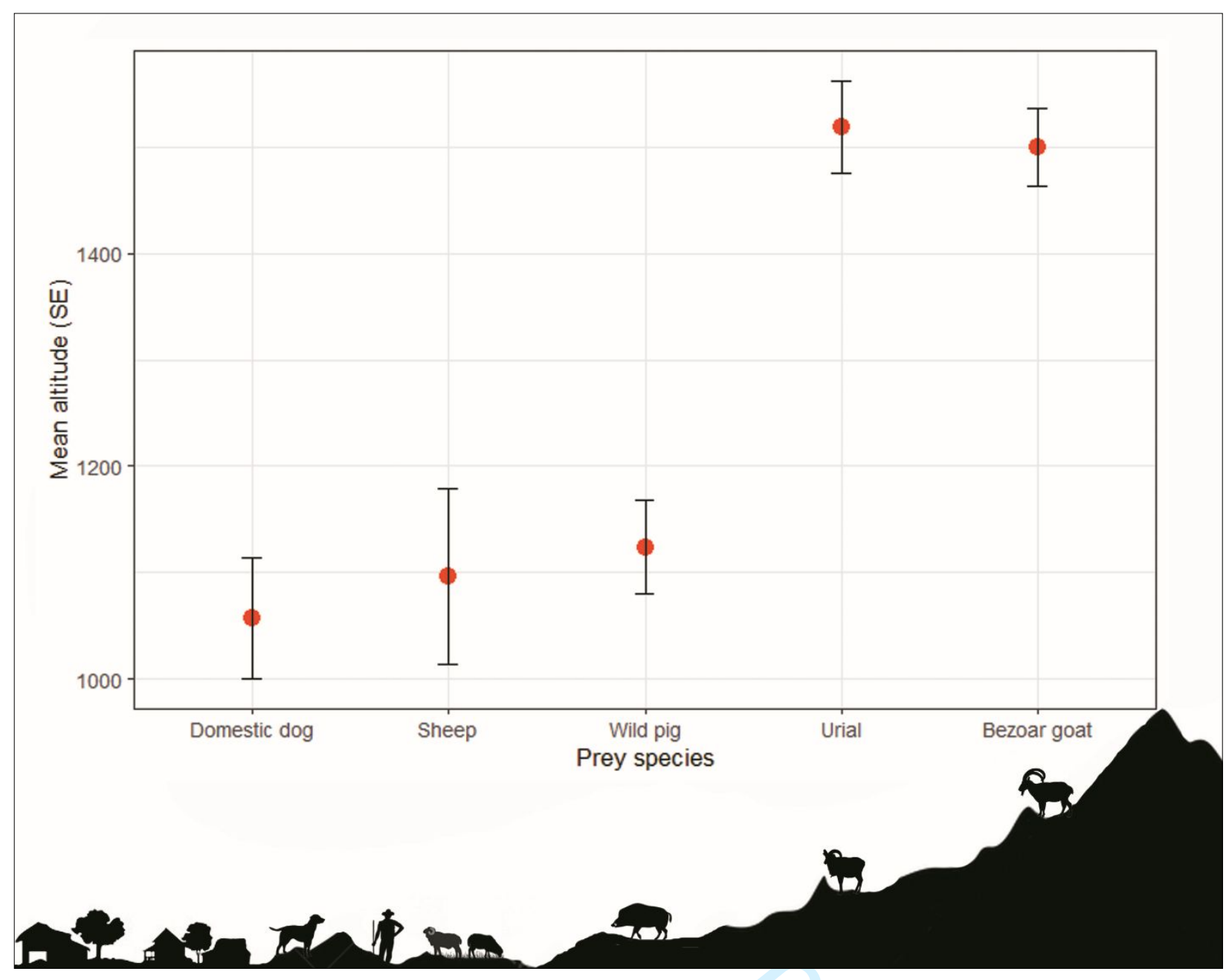

531

\section{$532 \quad$ Figure 4}

533 Altitudinal variability in kills, belonging to five main prey species, made by the collared Persian

534 leopards in Tandoureh National Park (2014-2017). Ranges on the graphic are not of a scale 535 relative to each other. Error bars represent standard errors. 